\title{
Therapeutic effect of hybrid training of voluntary and electrical muscle contractions in middle-aged obese women with nonalcoholic fatty liver disease: a pilot trial
}

\author{
This article was published in the following Dove Press journal: \\ Therapeutics and Clinical Risk Management \\ 4 March 2015 \\ Number of times this article has been viewed
}

\section{Sechang $O h^{1,4}$ \\ Tsuyoshi Maruyama ${ }^{2}$ \\ Kiyoshi Eguchi² \\ Takashi Shida' \\ Emi Arai' \\ Tomonori Isobe' \\ Yoshikazu Okamoto ${ }^{3}$ \\ Junichi Shoda}

'Division of Medical Science, Faculty of Medicine, University of Tsukuba, Tsukuba, Ibaraki, Japan; ${ }^{2}$ Division of Rehabilitation, ${ }^{3}$ Division of Diagnostic Radiology, University of Tsukuba Hospital, Tsukuba, Ibaraki, Japan; ${ }^{4}$ Japan Society for the Promotion of Science, Tokyo, Japan
Correspondence: Junichi Shoda Division of Medical Science, Faculty of Medicine, University of Tsukuba, I-I-I Tennodai, Tsukuba, Ibaraki 305-8575, Japan

Tel +8I 298535795

Fax +8I 298535795

Email shodaj@md.tsukuba.ac.jp
Background: Exercise training is an effective therapy for nonalcoholic fatty liver disease (NAFLD). Hybrid training (HYB) of voluntary and electrical muscle contractions was developed to prevent disuse atrophy during space flight. HYB can be applied to obtain a strength training effect accompanying articular movement. In this pilot study, we aimed to investigate the therapeutic efficacy of HYB in NAFLD.

Methods: A total of 15 middle-aged obese women with NAFLD who had no improvement in serum alanine aminotransferase levels and/or liver fat deposition after 12 weeks of lifestyle counseling participated in an HYB program. HYB of the quadriceps and hamstrings was conducted for 20 minutes twice a week for 24 weeks.

Results: NAFLD patients showed attenuated intramyocellular lipid levels in the quadriceps after the HYB intervention (-15.5\%). Levels of leptin $(-17.4 \%)$, tumor necrosis factor- $\alpha(-23.2 \%)$, and interleukin-6 $(-30.5 \%)$ were also decreased after the intervention. HYB led to a significant body weight reduction $(-4.7 \%)$, which in turn was associated with a significant decrease in serum alanine aminotransferase $(-35.8 \%)$, gamma-glutamyl transpeptidase $(-21.6 \%)$, ferritin $(-16.0 \%)$, oxidative stress $(-17.8 \%)$ levels, and insulin resistance values $(-2.7 \%)$.

Conclusion: In NAFLD, HYB exerts an antiobesity effect and attenuates liver dysfunction and insulin resistance in association with an increase in muscle strength and a decrease in ectopic muscle fat. Therefore, HYB has great potential as a new type of exercise therapy for liver disease in patients with NAFLD.

Keywords: exercise, muscle, nonalcoholic fatty liver disease, obesity, oxidative stress, ectopic fat

\section{Introduction}

With sedentary lifestyles and imbalanced dietary choices, the obese population is increasing in Japan. ${ }^{1}$ This growing obesity epidemic increases the risk of mortality and morbidity, which is a major public health concern in Japan. In 2012, the Japan Society of Ningen Dock reported that liver dysfunction is the single most frequent abnormal finding in multiphase health screening. ${ }^{2}$ The main cause of liver dysfunction is considered to be nonalcoholic fatty liver disease (NAFLD), a condition characterized by excessive accumulation of hepatocellular lipids, ie, steatosis, and is strongly associated with obesity. ${ }^{3}$

Other than dietary and exercise therapies, no effective treatment for NAFLD has been identified. ${ }^{4}$ Although the importance of exercise in NAFLD is widely recognized, people often have difficulty finding sufficient time to exercise. Meanwhile, obese 
patients often find exercise difficult to perform because of the excess weight exerted on the knee and hip joints.

To improve the quality of life of obese subjects with NAFLD, it is important to maintain their skeletal muscle mass through moderate exercise. ${ }^{5}$ Skeletal muscles are not merely organs of locomotion; as metabolic organs, they are also involved in regulation of glucose metabolism. ${ }^{6}$ In particular, in cases where the patient is middle-aged or older, finding ways to prevent age-related muscle atrophy and decreased muscle strength while improving metabolic function is important for preventing the spread of liver lesions. Under such circumstances, it is necessary for those with NAFLD to practice an exercise training method that is simple, safe, and effective.

Hybrid training (HYB) was developed to prevent muscle atrophy in astronauts spending long periods in space stations. ${ }^{7}$ In the present study, we used HYB as a form of exercise therapy for NAFLD. HYB is a type of skeletal muscle training that combines spontaneous muscular constriction with electrical constriction in response to electrical stimulation. The contractile force generated by applying an electrical stimulus to the antagonist opposing the agonist muscle is utilized as motion resistance. These principles can be applied to obtain a strength training effect accompanying articular movement. In addition, HYB has a greater training effect than conventional electrical training methods because it uses centrifugal contraction induced by electrical stimulation, which makes it possible to utilize a greater exercise load with the same amount of electrical stimulation. The intensity of the electrical stimulation can be adjusted, making it possible to minimize the pain and discomfort of the electrical stimulus. ${ }^{8-10} \mathrm{HYB}$ can be performed over a short period of time in a seated position, making it suitable for patients who are severely obese and those who have arthritis of the lumbar spine or knee.

Following on from recent studies, ${ }^{11,12}$ we conducted a pilot study involving middle-aged obese women with NAFLD who underwent HYB for 24 weeks to evaluate its efficacy from the standpoints of skeletal muscle mass, strength and ectopic adipose, body composition, glucose and lipid metabolism, liver dysfunction, and improvement in inflammatory and oxidative stress markers.

\section{Materials and methods Subjects and study design}

This study was conducted as a pilot to investigate the therapeutic efficacy of HYB for the lower extremities in middle-aged obese women with NAFLD. Figure 1 depicts the workflow of enrollment of study subjects. Between April 2011 and December 2014, 55 patients with NAFLD presented to an outpatient department specializing in lifestyle-related liver diseases at the Tsukuba University Hospital. None of the patients consumed excess alcohol ( $>20 \mathrm{~g} /$ day), had another cause of liver disease or a psychiatric illness, or were taking antidiabetic or weight loss agents. Each patient received lifestyle counseling regarding diet and physical activity for NAFLD according to the guidelines of the American Gastroenterological Association. ${ }^{13}$ The counseling was conducted by a registered dietitian and a medical doctor specializing in nutrition. Twelve weeks after the counseling, 40 patients showed either a decrease or no change in serum alanine aminotransferase (ALT) levels and liver fat deposition evaluated by abdominal ultrasonography. ${ }^{14}$ The remaining 15 patients (mean age 61.2 \pm 6.0 years) showed an increase in serum ALT levels or liver fat deposition and were enrolled in this study. These women with NAFLD visited the rehabilitation division of Tsukuba University Hospital twice a week and performed HYB. The diagnosis of NAFLD was based on elevated serum ALT levels and the presence of at least two of three abnormal findings on abdominal ultrasonography, ie, diffusely increased liver echogenicity ("bright") that was greater than for the kidney, vascular blurring, or deep attenuation of the ultrasound signal according to the diagnostic guidelines for NAFLD in the Asia-Pacific region. ${ }^{15}$

Written informed consent was obtained from each patient. The study protocol conformed to the ethical guidelines of the 1975 Declaration of Helsinki, as reflected in prior approval by the ethics committee of the University of Tsukuba Faculty of Medicine.

\section{Hybrid training}

HYB was performed in a sitting position without the feet touching the floor, as previously described. ${ }^{9,10}$ Briefly, the hamstrings were electrically stimulated when the knee was voluntarily extended (Figure 2A), whereas the quadriceps

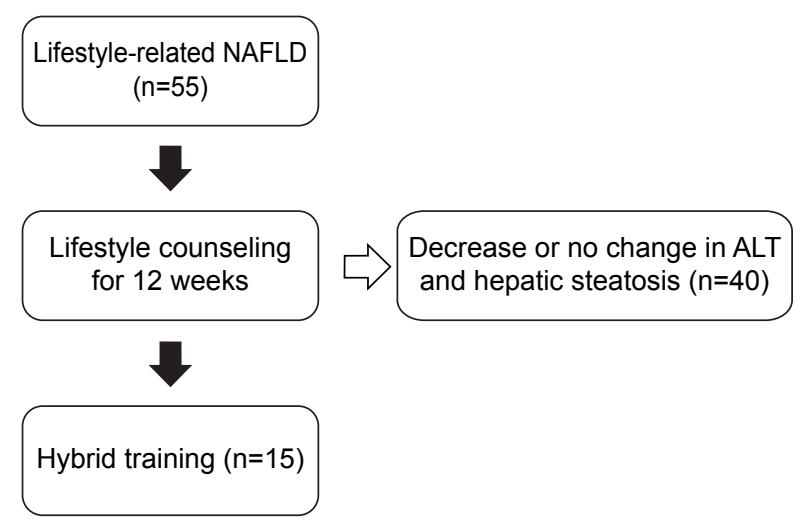

Figure I Enrollment of study subjects. Abbreviations: ALT, alanine aminotransferase; NAFLD, nonalcoholic fatty liver disease. 

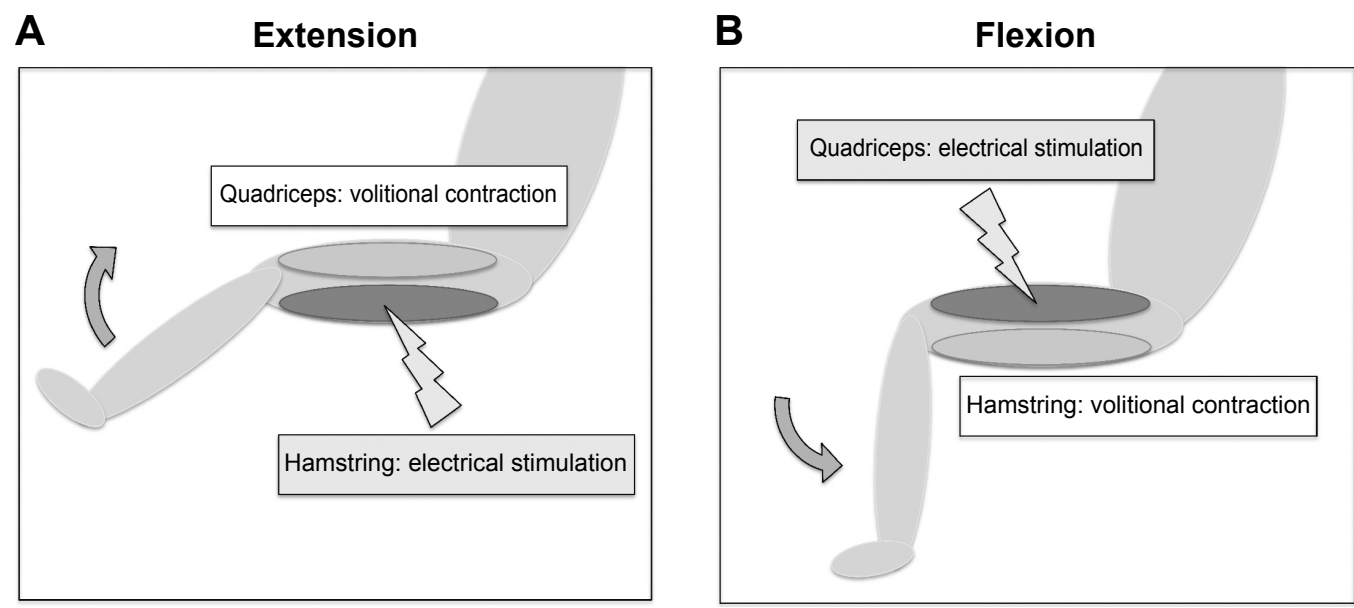

Figure 2 Principle of hybrid training.

Notes: During knee extension (A), the quadriceps are voluntarily contracted and the hamstrings are electrically stimulated at the same time. During knee flexion (B), the hamstrings are voluntarily contracted and the quadriceps are electrically stimulated at the same time.

were electrically stimulated when the knee was voluntarily bent and flexed (Figure 2B).

The HYB lasted for 19 minutes per session, and sessions were scheduled twice a week for 24 weeks (a total of 48 sessions). Each session consisted of ten sets of ten reciprocal 3 -second knee flexion and extension contractions. The sets were separated by a one-minute rest interval. The electrical stimulation intensity was set at a level of 20-25 consecutive knee flexions and extensions. The mean stimulating voltages were $29.5 \pm 6.5 \mathrm{~V}$ and $31.4 \pm 6.7 \mathrm{~V}$ for the quadriceps and hamstring muscles, respectively. This voltage level does not cause pain. The joint motion sensor (Mutoh Engineering Inc., Tokyo, Japan) triggered stimulation of the antagonist muscle once it sensed initiation of volitional contraction of the agonist.

\section{Assessment of total energy intake}

For assessment of total daily energy intake, the subjects recorded their food intake for 3 consecutive days at both baseline and week 24 under the supervision of a professional dietitian. The dietitian estimated these using Excel Eiyo-Kun version 4 software (Kenpakusya, Tokyo, Japan).

\section{Body weight and composition}

Body weight and composition were measured using an InBody720 (Biospace Japan Inc., Tokyo, Japan). Standing height was measured by a wall-mounted stadiometer (Muratec-KDS Co., Kyoto, Japan) and used to calculate body mass index $\left(\mathrm{kg} / \mathrm{m}^{2}\right)$.

\section{Measurement of muscle strength}

Maximal strength of isometric contraction of knee flexion and extension at an angle of $90^{\circ}$ in a seated position was measured using a dynamometer (Biodex III, Biodex Medical Systems Inc., Shirley, NY, USA). The peak torque (Nm) of the records of three consecutive trials was considered as the maximal strength. Each isometric contraction was held for 3 seconds. The results were normalized by body weight $(\mathrm{kg})$.

\section{Ultrasonography}

An ultrasound scanner (SSD-5500, Aloka Co. Ltd., Tokyo, Japan) was used to evaluate steatosis of the liver. The grade of liver steatosis was determined by a clinical radiologist according to the criteria of Saadeh et al. ${ }^{14}$ The radiologist was blinded to treatment allocation. The films were scored in random order without any clinical information.

\section{Magnetic resonance spectroscopy}

A 3-Tesla magnetic resonance device (Achieva, Philips Electronics Japan, Ltd., Tokyo, Japan) equipped with a 6-channel Torso coil was used for this study. The excitation method was spin echo point-resolved spatially localized spectroscopy, and data were acquired by the single voxel method. The data obtained were analyzed with LCModel software (LA Systems Inc., Tokyo, Japan). ${ }^{16}$

\section{Laboratory determinations}

Blood samples were drawn from the median cubital vein at baseline and at week 24 following a period of no training for 48 hours and fasting for 12 hours. Triglyceride and high-density lipoprotein cholesterol levels were analyzed by an enzyme method, aspartate aminotransferase (AST), ALT, and gamma-glutamyl transpeptidase $(\gamma-\mathrm{GT})$ levels by the transferable method of the Japan Society of Clinical Chemistry, fasting plasma insulin level by a chemiluminescent 
immunoassay method, high-sensitivity C-reactive protein by a fixed time assay method, fasting plasma glucose by a glucose-6-phosphate dehydrogenase method, and glycated hemoglobin, hyaluronic acid, plasma type IV collagen, and ferritin levels by a latex agglutination method. Commercial enzyme-linked immunosorbent assay kits were used to measure serum levels of total adiponectin (Sekisui Medical Co., Ltd., Tokyo, Japan), tumor necrosis factor-alpha (TNF- $\alpha$ ), interleukin (IL)-6, and leptin (R\&D Systems Inc., Minneapolis, MN, USA), and thiobarbituric acid reactive substances (Cayman Chemical Co., Ann Arbor, MI, USA). Surrogate markers were calculated using the homeostasis model assessment of insulin resistance (HOMA-IR) ${ }^{17}$ and from NAFLD fibrosis scores. ${ }^{18}$

\section{Statistical analysis}

The statistical analysis was performed using Statistical Package for the Social Sciences version 20 software (SPSS Inc, Chicago, IL, USA). Descriptive parameters show the median ( $95 \%$ confidence interval) or proportion $(\%)$. Effects of training were evaluated by changes (baseline-after 24 weeks) in the variable and were analyzed using the Wilcoxon signed-rank test.

\section{Results}

\section{Adherence to and adverse events of hybrid training program}

None of the patients developed any NAFLD-related complications during the study period, and all completed the study. During the HYB program, no severe adverse events developed, including electric stimulation-related burns or muscle strain, although muscle fatigue was seen in all patients. Based on the 3-day food records, the total estimated mean ( \pm standard deviation) daily energy intake from baseline to week 24 was $1,513 \pm 151 \mathrm{kcal} /$ day to $1,573 \pm 205 \mathrm{kcal} /$ day.
Effects of hybrid training on body weight, skeletal muscle, and fat

Body weight $(-4.7 \%)$ and body fat $(-3.8 \%)$ were significantly decreased after the HYB intervention (Table 1). However, skeletal muscle $(-1.9 \%)$ and waist circumference $(-2.3 \%)$ were not significantly changed after the intervention (Table 1).

\section{Effects of hybrid training on muscle strength and muscle fat deposition}

Although no significant changes in skeletal muscle mass were observed (Figure 3A), muscle strength of the quadriceps $(+18.4 \%)$, but not that of the hamstrings $(+12.5 \%)$, was significantly increased after the intervention, as shown in Figure 3B. On analysis by ${ }^{1} \mathrm{H}$ nuclear magnetic resonance spectroscopy, levels of intramyocellular lipids $(-15.5 \%)$ but not extramyocellular lipids $(-7.3 \%)$ were decreased in the quadriceps after HYB (Figure 4).

\section{Effects of hybrid training on liver steatosis}

Liver steatosis grade, determined by ultrasonography, was significantly decreased after HYB (-16.7\%), as shown in Table 2.

\section{Effects of hybrid training on liver dysfunction}

Liver function tests revealed significant improvements in serum AST (-18.4\%), ALT (-35.8\%), and $\gamma$-GT (-21.6\%) levels after the HYB intervention (Table 2).

\section{Effects of hybrid training on lipid profile}

There was a significant decrease in serum free fatty acid levels $(-4.9 \%)$ after the HYB intervention (Table 2$)$. However, other lipids, including high-density lipoprotein cholesterol $(-2.8 \%)$, low-density lipoprotein cholesterol $(-6.5 \%)$, and triglycerides $(-6.4 \%)$, were not significantly different after the intervention (Table 2).

Table I Outcome for body weight and composition in 15 middle-aged obese women with nonalcoholic fatty liver disease who participated in a hybrid training program

\begin{tabular}{llll}
\hline Parameters & Hybrid training $(\mathbf{n}=\mathbf{1 5})$ & Change \\
\cline { 2 - 3 } & Pre treatment & Post treatment & \\
\hline Anthropometric and body composition & & $60.3(58.6-70.4)$ & $-3.0 *$ \\
$\quad$ Weight $(\mathrm{kg})$ & $63.3(57.7-71.8)$ & $20.7(19.8-21.4)$ & -0.4 \\
$\quad$ Skeletal muscle $(\mathrm{kg})$ & $21.1(19.8-21.9)$ & $22.6(20.5-31.3)$ & $-0.9 *$ \\
Body fat $(\mathrm{kg})$ & $23.5(20.6-31.0)$ & $89.2(82.6-101.0)$ & -2.1 \\
$\quad$ Waist circumference $(\mathrm{cm})$ & $91.3(86.6-101.5)$ &
\end{tabular}

Notes: Values are presented as the group medians ( $95 \%$ confidence interval). The comparison of changes over time, from pre treatment to post treatment, for all dependent variables was analyzed by Wilcoxon's rank sum test. $* P<0.05$, significant difference between baseline and week 24 . 
A

Quadriceps

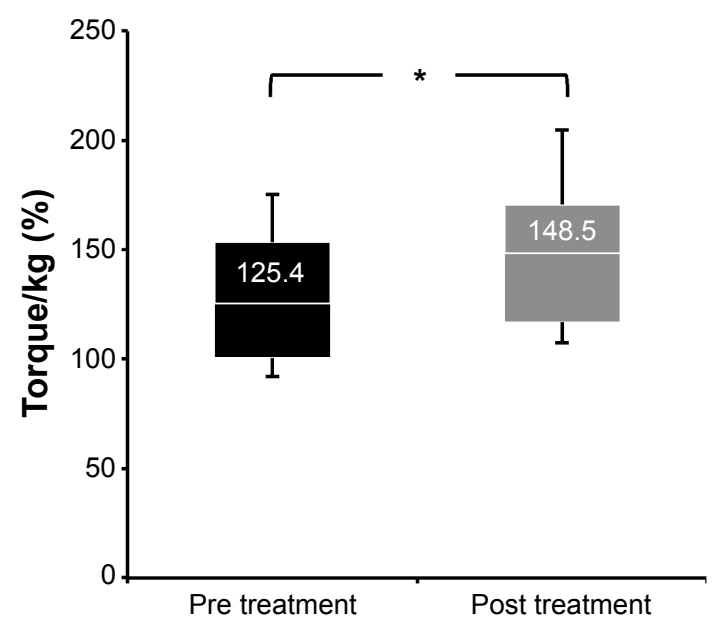

B

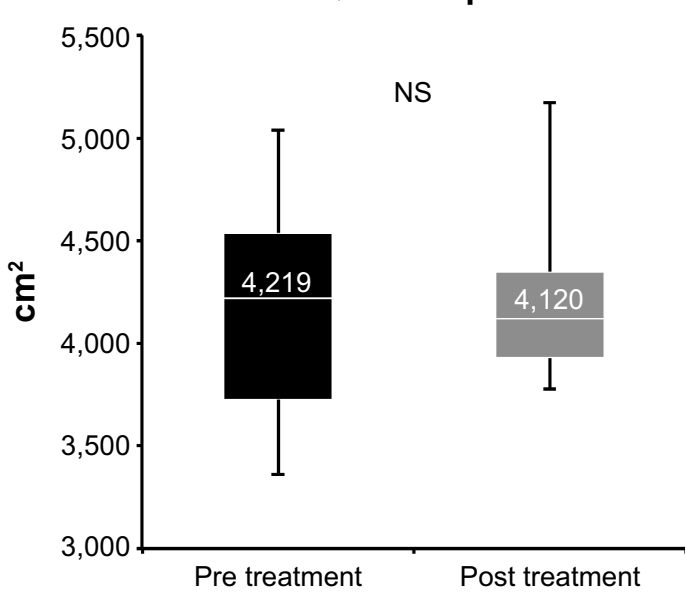

Hamstrings

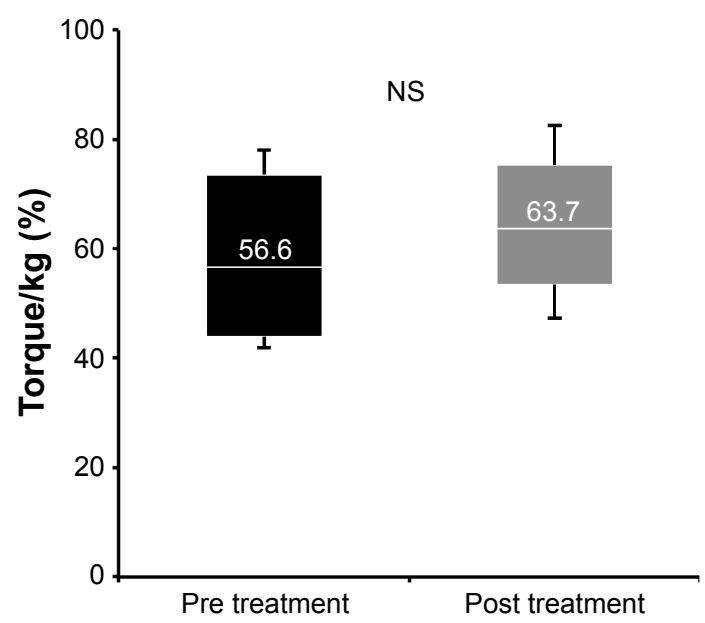

Hamstrings

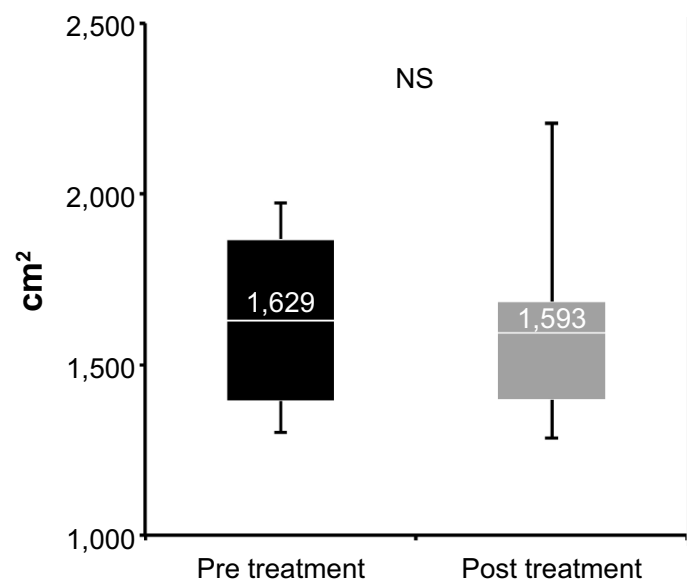

Figure 3 Effects of the hybrid training on the muscle cross-sectional area $(\mathbf{A})$, strength $(\mathbf{B})$ of the femurs of middle-aged obese women with nonalcoholic fatty liver disease. $* P<0.05$, significant difference between baseline and week 24 .

Notes: The lower edge, midline, and upper edge of each box represent the 25 th percentile, median, and 75 th percentile scores, respectively. The line from each box extends to the minimum and maximum score.

Abbreviation: NS, not statistically significant.

\section{Effects of hybrid training on insulin resistance}

Fasting plasma glucose $(-0.8 \%)$ and HOMA-IR (-2.7\%) were significantly decreased after the HYB intervention. However, fasting plasma insulin $(-10.6 \%)$ and glycated hemoglobin $(-3.1 \%)$ were not significantly decreased (Table 2).

\section{Effects of hybrid training on adipocytokine levels}

The participants showed a significant reduction in serum leptin (-17.4\%), TNF- $\alpha(-23.2 \%)$, and IL-6 (-30.5\%) levels after the HYB intervention. However, adiponectin levels $(+10.8 \%)$ were not significantly increased (Figure 5$)$.

\section{Effects of hybrid training on inflammatory, oxidative stress, and hepatic fibrosis markers}

Inflammatory markers, the high-sensitivity C-reactive protein $(-36.1 \%)$ and ferritin $(-16.0 \%)$, and thiobarbituric acid reactive substances $(-17.8 \%)$ as known oxidative stress marker levels were significantly decreased after the HYB intervention (Table 2). Liver fibrosis markers, eg, hyaluronic acid $(+35.3 \%)$ and type IV collagen $(-12.8 \%)$ levels, and the NAFLD fibrosis score $(-107.6 \%)$ were not significantly improved (Table 2$)$.

\section{Discussion}

The present study shows that HYB in middle-aged obese women with NAFLD increased skeletal muscle strength in 
IMCL

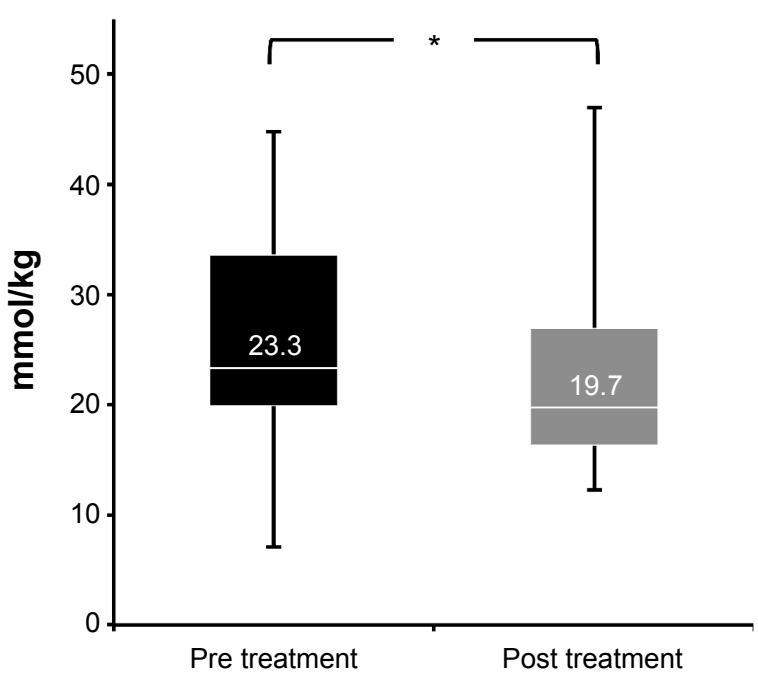

\section{EMCL}

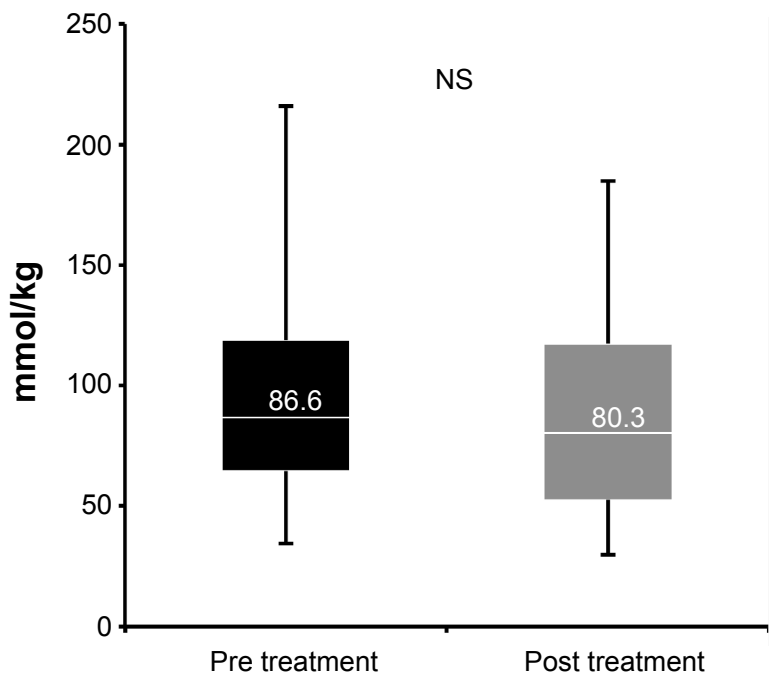

Figure 4 Effects of hybrid training on muscle fat deposit in the femurs of middle-aged obese women with nonalcoholic fatty liver disease. $* P<0.05$, significant difference between baseline and week 24 .

Notes: The lower edge, midline, and upper edge of each box represent the 25 th percentile, median, and 75 th percentile scores, respectively. The line from each box extends to the minimum and maximum score.

Abbreviations: EMCL, extramyocellular lipids; IMCL, intramyocellular lipids; NS, not statistically significant.

Table 2 Outcomes for hepatic steatosis and blood testing in 15 middle-aged obese women with nonalcoholic fatty liver disease who participated in a hybrid training program

\begin{tabular}{|c|c|c|c|}
\hline \multirow[t]{2}{*}{ Parameters } & \multicolumn{2}{|c|}{ Hybrid training $(n=15)$} & \multirow[t]{2}{*}{ Change } \\
\hline & Pre treatment & Post treatment & \\
\hline \multicolumn{4}{|l|}{ Ultrasonography } \\
\hline Hepatic steatosis grade & $3.0(2.0-3.0)$ & $2.5(1.0-3.0)$ & $-0.5^{*}$ \\
\hline \multicolumn{4}{|l|}{ Liver dysfunction } \\
\hline AST (U/L) & $38(23-7 I)$ & $3 \mid(2 \mid-5 I)$ & $-7^{*}$ \\
\hline $\mathrm{ALT}(\mathrm{U} / \mathrm{L})$ & $53(20-65)$ & $34(22-54)$ & $-19 *$ \\
\hline$\gamma$-GT (U/L) & $37(19-62)$ & $29(18-49)$ & $-8^{*}$ \\
\hline \multicolumn{4}{|l|}{ Lipid profile } \\
\hline FFA (mEq/L) & $0.81(0.63-1.01)$ & $0.77(0.50-0.91)$ & $-0.04 *$ \\
\hline TG (mg/dL) & $110(73-152)$ & $103(79-128)$ & -7 \\
\hline HDL-C (mg/dL) & $54.4(49.9-59.9)$ & $52.9(46.6-60.8)$ & -1.5 \\
\hline LDL-C (mg/dL) & $124(100-145)$ & $116(101-140)$ & -8 \\
\hline \multicolumn{4}{|l|}{ Insulin resistance } \\
\hline FPG (mg/dL) & $123(1 \mid 1-173)$ & $122(108-145)$ & $-I^{*}$ \\
\hline $\mathrm{FPI}(\mu \mathrm{U} / \mathrm{mL})$ & II.3 (7.5-15.9) & $10.1(6.7-16.3)$ & -1.2 \\
\hline HOMA-IR & $3.39(2.26-6.04)$ & $3.30(1.77-5.27)$ & $-0.09 *$ \\
\hline $\mathrm{HbA}_{\mathrm{Ic}}(\%)$ & $6.4(5.5-7.0)$ & $6.2(5.3-7.1)$ & -0.2 \\
\hline \multicolumn{4}{|c|}{ Inflammatory and oxidative stress } \\
\hline hs-CRP (mg/dL) & $0.097(0.034-0.228)$ & $0.062(0.033-0.165)$ & $-0.035^{*}$ \\
\hline Ferritin (ng/mL) & $125(5|-| 58)$ & $105(46-156)$ & $-20 *$ \\
\hline TBARS $(\mu \mathrm{M})$ & $19.7(16.3-26.2)$ & $16.2(15.1-19.0)$ & $-3.5^{*}$ \\
\hline \multicolumn{4}{|l|}{ Fibrosis markers } \\
\hline $\mathrm{HA}(\mathrm{ng} / \mathrm{mL})$ & $51(39-69)$ & $69(35-85)$ & +18 \\
\hline Type IV (ng/mL) & $187(130-216)$ & $163(133-202)$ & -24 \\
\hline NAFLD fibrosis score & $-0.157(-0.94 I, 0.627)$ & $-0.326(-0.870,0.219)$ & -0.169 \\
\hline
\end{tabular}

Notes: Values are presented as the group medians ( $95 \%$ confidence interval). The comparison of changes over time, from pre treatment to post treatment, for all dependent variables was analyzed by Wilcoxon's rank sum test. $* P<0.05$, significant difference between the baseline and the 24th week.

Abbreviations: AST, aspartate aminotransferase; ALT, alanine aminotransferase; $\gamma$-GT, gamma-glutamyl transpeptidase; FFA, free fatty acid; TG, triglycerides; HDL-C, highdensity lipoprotein cholesterol; LDL-C, low-density lipoprotein cholesterol; FPG, fasting plasma glucose; FPI, fasting plasma insulin; $\mathrm{HbA}_{\mathrm{Ic}}$, glycated hemoglobin; HOMA-IR, homeostasis model assessment of insulin resistance; hs-CRP, high sensitivity C-reactive protein; TBARS, thiobarbituric acid reactive substances; HA, hyaluronic acid; NAFLD, nonalcoholic fatty liver disease; Type IV, type IV collagen. 

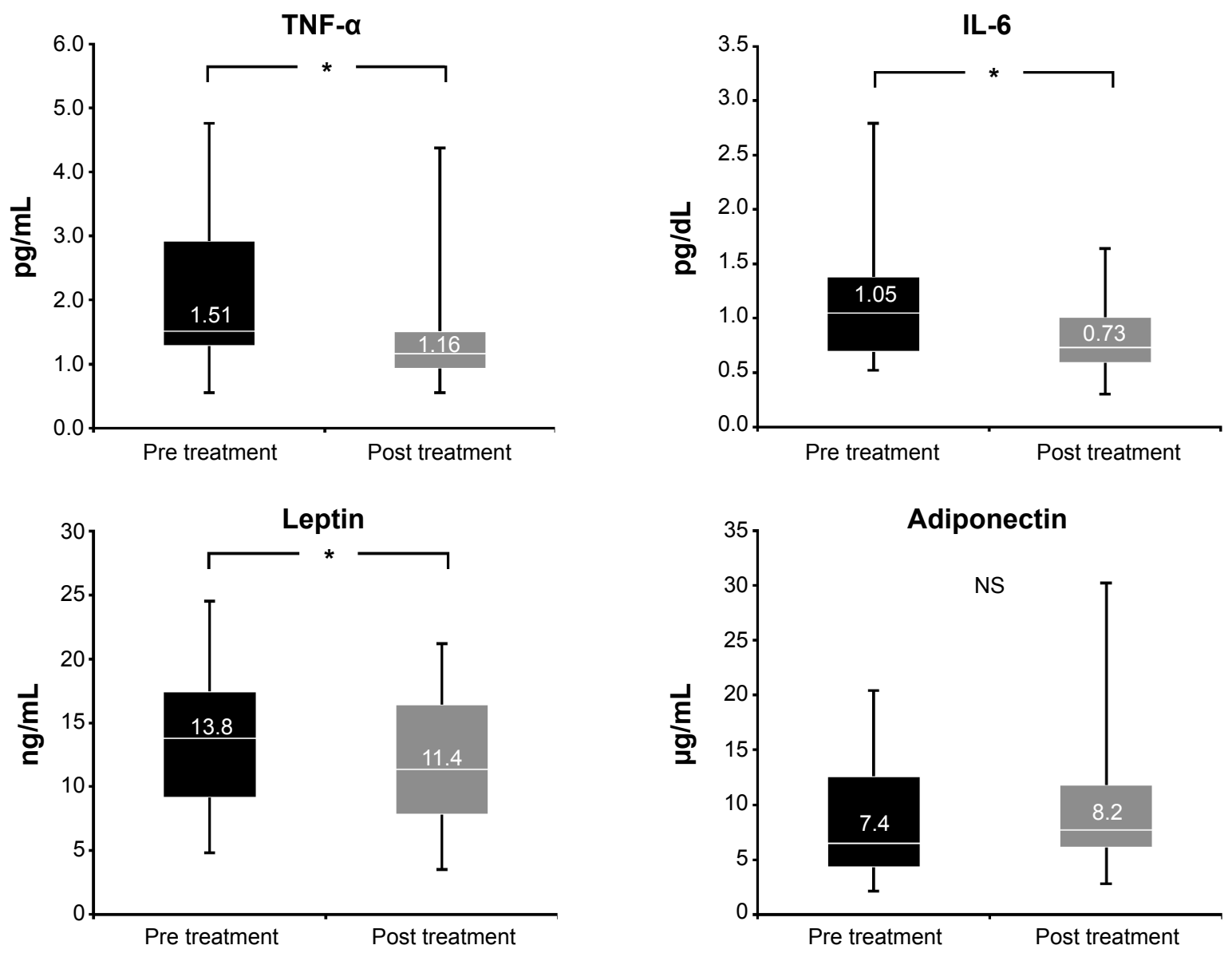

Figure 5 Effects of hybrid training on serum levels of TNF- $\alpha$, IL-6, leptin, and adiponectin in middle-aged obese women with nonalcoholic fatty liver disease. $* P<0.05$, significant difference between baseline and week 24 .

Notes: The lower edge, midline, and upper edge of each box represent the 25 th percentile, median, and 75 th percentile scores, respectively. The line from each box extends to the minimum and maximum score.

Abbreviations: IL-6, interleukin-6; NS, not statistically significant; TNF- $\alpha$, tumor necrosis factor-alpha.

those parts of the body that received the training. Moreover, HYB decreased intramyocellular lipid concentrations, reduced body fat mass, and attenuated liver steatosis. It should be noted that HYB achieved a significantly greater degree of attenuation in the pathological indices of insulin resistance and liver dysfunction.

As with other types of resistance training, HYB was not found to be superior to the control condition in terms of improving body composition in obese women with NAFLD. The improvement effect of this training with regard to body fat mass is consistent with a previous report indicating low effectiveness of ordinary electrical muscle stimulation for decreasing body fat mass. ${ }^{19}$ However, the decrease in body fat mass observed after training is a factor that explains the significant decrease in blood leptin level. Leptin is an important factor promoting liver fibrosis in the pathogenesis of NAFLD. ${ }^{20}$

One of the advantages of HYB is that it decreases blood liver enzyme (ALT, AST, and $\gamma$-GT) levels, thereby alleviating liver dysfunction and improving the condition of the liver in patients with NAFLD. However, our findings were inadequate to suggest that the change in body composition resulting from HYB was responsible for the attenuated liver dysfunction. The presence of factors such as insulin resistance ${ }^{21}$ and oxidative stress ${ }^{22,23}$ is important in the spread of hepatic lesions. The underlying mechanism of improvement in the state of the liver in patients with NAFLD who receive HYB may be assumed to involve attenuation of insulin resistance that is not mediated by a decrease in body weight, together with induction of molecular mechanisms involved in activation of the mechanism of endogenous response to oxidative stress.

The post-training attenuation of levels of ferritin, an inflammatory marker, the tendency toward attenuation in high-sensitivity C-reactive protein levels, and attenuation of serum levels of leptin, an adipokine, and of proinflammatory cytokines, eg, TNF- $\alpha$ and IL-6, suggest that the underlying mechanism of improvement of liver dysfunction brought about by HYB may involve attenuation of oxidative stress. 


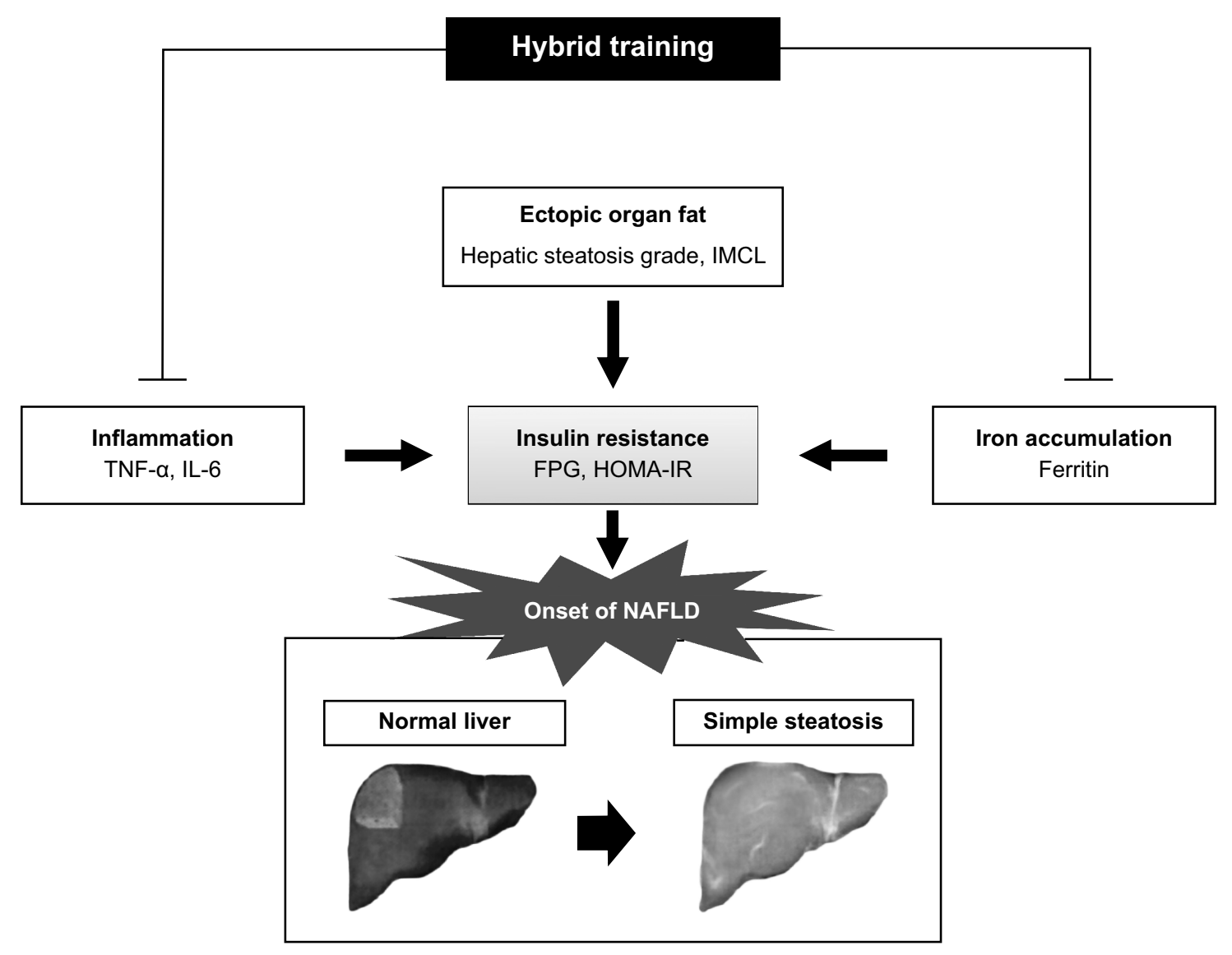

Figure 6 Schematic summary of the beneficial effects of the hybrid training in middle-aged obese women with NAFLD.

Abbreviations: FPG, fasting plasma glucose; HOMA-IR, homeostasis model assessment of insulin resistance; IL-6, interleukin-6; IMCL, intramyocellular lipids; NAFLD, nonalcoholic fatty liver disease; TNF- $\alpha$, tumor necrosis factor-alpha.

Another point of superiority of HYB with respect to skeletal muscle is that an increase in the strength of quadriceps femoris and a decrease in intramyocellular lipids were observed in the absence of an increase in skeletal muscle mass (Figures 3 and 4). In addition to intrahepatic fat content, fatty metamorphosis of skeletal muscle tissue has been shown to contribute independently to the onset of insulin resistance. ${ }^{24}$ The decrease in intramyocellular lipid levels resulting from HYB was considered to be related to attenuation of HOMA-IR. Moreover, the decreases in blood levels of free fatty acids, leptin, TNF- $\alpha$, and IL- 6 resulting from HYB were considered to be factors contributing to the attenuation of insulin resistance. Leptin acts via the sympathetic nervous system to induce lipolysis ${ }^{25}$ and known good predictors to detect the presence of liver steatosis. ${ }^{26}$ Improvement of liver dysfunction by HYB is considered to be the consequence of restoration of insulin sensitivity and reduction of abnormal fat accumulation in the liver via resolution of the adipokine imbalance (Figure 6).
Interestingly, "exercise factors" known as myokines ${ }^{27}$ may play a part in the improvement elicited by HYB in patients with NAFLD. Myokines are released by skeletal muscle cells during contraction and have endocrine activity. Research findings suggest that IL-6 is a molecule that triggers exercise-induced metabolic changes in the liver and adipose tissue. ${ }^{28}$ In addition, exercise has been reported to decrease IL-6 transcription in skeletal muscle. ${ }^{29}$ However, future study of the relationship between myokines and skeletal muscle training at a more detailed molecular level is needed.

The guidelines for management of nonalcoholic steatohepatitis/NAFLD published by the Japan Society of Hepatology ${ }^{30}$ discuss exercise therapy and describe methods similar to those recommended for obesity. ${ }^{31}$ Although not with specific reference to NAFLD, a minimum of 20 minutes of aerobic exercise a day is recommended. In the guidelines, anaerobic exercise (eg, short-range sprinting, weight lifting, and push-ups) is not recommended. Nevertheless, our results for HYB, albeit from a pilot trial, indicate that the effects 
of strength training as resistance exercise is of considerable significance because, although the effect in terms of weight reduction is small, metabolic function can be improved while maintaining skeletal muscle mass. As resistance exercise, HYB reduces ectopic steatosis in the liver and the skeletal muscles while attenuating insulin resistance, which is extremely useful in the management of patients with NAFLD.

In conclusion, the present study demonstrates that HYB for the lower extremities in middle-aged obese women with NAFLD exerts an antiobesity effect and attenuates liver dysfunction and insulin resistance in association with an increase in muscle strength and a decrease in ectopic muscle fat. Therefore, HYB has potential as a new type of exercise therapy for liver disease in patients with NAFLD.

\section{Acknowledgments}

We thank Professor Naoto Shiba, Department of Orthopedics, Kurume University Hospital, for his collaboration in the design of the training program and his excellent technical assistance therein. This work was supported in part by Grants-in-Aid for Scientific Research from the Ministry of Education, Culture, Sports, Science, and Technology, Japan (24390488, 25282172, 25560364, 26293284, 26282191, 26293297, and 26670109).

\section{Disclosure}

No commercial party having a direct financial interest in the results of the research supporting this article has or will confer a benefit on the authors or on any organization with which the authors are associated. The authors report no conflicts of interest in this work.

\section{References}

1. Eguchi E, Iso H, Tanabe N, et al. Healthy lifestyle behaviours and cardiovascular mortality among Japanese men and women: the Japan Collaborative Cohort Study. Eur Heart J. 2012;33(4):467-477.

2. The Japan Society of Ningen Dock. Present status of Ningen Dock in 2012. The Annual Report on Japan Society of Ningen Dock. Available from: http://www.ningen-dock.jp/wp/common/data/other/release/dockgenkyou_h24.pdf. Accessed December 7, 2014. Japanese.

3. Farrell GC, Larter CZ. Nonalcoholic fatty liver disease: from steatosis to cirrhosis. Hepatology. 2006;43(2):S99-S112.

4. Centis E, Marzocchi R, Suppini A, et al. The role of lifestyle change in the prevention and treatment of NAFLD. Curr Pharm Des. 2013; 19(29):5270-5279.

5. Finelli C, Tarantino G. Have guidelines addressing physical activity been established in nonalcoholic fatty liver disease? World J Gastroenterol. 2012;18(46):6790-6800.

6. Jensen J, Rustad PI, Kolnes AJ, Lai YC. The role of skeletal muscle glycogen breakdown for regulation of insulin sensitivity by exercise. Front Physiol. 2011;2:112.
7. Yanagi T, Shiba N, Maeda T, et al. Agonist contractions against electrically stimulated antagonists. Arch Phys Med Rehabil. 2003;84(6):843-848.

8. Matsuse H, Shiba N, Umezu Y, et al. Muscle training by means of combined electrical stimulation and volitional contraction. Aviat Space Environ Med. 2006;77(6):581-585.

9. Iwasaki T, Shiba N, Matsuse H, et al. Improvement in knee extension strength through training by means of combined electrical stimulation and voluntary muscle contraction. Tohoku J Exp Med. 2006; 209(1):33-40.

10. Takano Y, Haneda Y, Maeda T, et al. Increasing muscle strength and mass of thigh in elderly people with the hybrid-training method of electrical stimulation and volitional contraction. Tohoku J Exp Med. 2010; 221(1):77-85.

11. Kawaguchi T, Shiba N, Takano Y, Maeda T, Sata M. Hybrid training of voluntary and electrical muscle contractions decreased fasting blood glucose and serum interleukin-6 levels in elderly people: a pilot study. Appl Physiol Nutr Metab. 2011;36(2):276-283.

12. Kawaguchi $T$, Shiba N, Maeda $T$, et al. Hybrid training of voluntary and electrical muscle contractions reduces steatosis, insulin resistance, and il-6 levels in patients with NAFLD: a pilot study. J Gastroenterol. 2011; 46(6):746-757.

13. American Gastroenterological Association. American Gastroenterological Association Medical Position Statement: nonalcoholic fatty liver disease. Gastroenterology. 2002;123(5):1702-1704.

14. Saadeh S, Younossi ZM, Remer EM, et al. The utility of radiological imaging in nonalcoholic fatty liver disease. Gastroenterology. 2002; 123(3):745-750.

15. Farrell GC, Chitturi S, Lau GK, Sollano JD. Guidelines for the assessment and management of non-alcoholic fatty liver disease in the Asia-Pacific region: executive summary. J Gastroenterol Hepatol. 2007; 22(6):775-777.

16. Provencher SW. Estimation of metabolite concentrations from localized in vivo proton NMR spectra. Magnetic resonance in medicine. Magn Reson Med. 1993;30(6):672-679.

17. Matthews DR, Hosker JP, Rudenski AS, Naylor BA, Treacher DF, Turner RC. Homeostasis model assessment: insulin resistance and betacell function from fasting plasma glucose and insulin concentrations in man. Diabetologia. 1985;28(7):412-419.

18. Angulo P, Hui JM, Marchesini G, et al. The NAFLD fibrosis score: a noninvasive system that identifies liver fibrosis in patients with NAFLD. Hepatology. 2007;45(4):846-854.

19. Porcari JP, McLean KP, Foster C, Kernozek T, Crenshaw B, Swenson C. Effects of electrical muscle stimulation on body composition, muscle strength, and physical appearance. J Strength Cond Res. 2002;16(2):165-172.

20. Ikejima $\mathrm{K}$, Takei $\mathrm{Y}$, Honda $\mathrm{H}$, et al. Leptin receptor-mediated signaling regulates hepatic fibrogenesis and remodeling of extracellular matrix in the rat. Gastroenterology. 2002;122(5):1399-1410.

21. Torres DM, Harrison SA. Diagnosis and therapy of nonalcoholic steatohepatitis. Gastroenterology. 2008;134(6):1682-1698.

22. Sumida Y, Niki E, Naito Y, Yoshikawa T. Involvement of free radicals and oxidative stress in NAFLD/NASH. Free Radic Res. 2013;47(11): 869-880.

23. Oh S, Tanaka K, Warabi E, Shoda J. Exercise reduces inflammation and oxidative stress in obesity-related liver diseases. Med Sci Sports Exerc. 2013;45(12):2214-2222.

24. Kitajima Y, Eguchi Y, Ishibashi E, et al. Age-related fat deposition in multifidus muscle could be a marker for nonalcoholic fatty liver disease. J Gastroenterol. 2010;45(2):218-224.

25. Pinkney JH, Coppack SW, Mohamed-Ali V. Effect of isoprenaline on plasma leptin and lipolysis in humans. Clin Endocrinol. 1998;48(4): 407-411.

26. Abenavoli L, Luigiano C, Guzzi PH, et al. Serum adipokine levels in overweight patients and their relationship with non-alcoholic fatty liver disease. Panminerva Med. 2014;56(2):189-193. 
27. Pedersen BK, Febbraio MA. Muscles, exercise and obesity: skeletal muscle as a secretory organ. Nat Rev Endocrinol. 2012;8(8):457-465.

28. Pedersen BK. Muscles and their myokines. J Exp Biol. 2011;214(2): 337-346.

29. Bruun JM, Helge JW, Richelsen B, Stallknecht B. Diet and exercise reduce low-grade inflammation and macrophage infiltration in adipose tissue but not in skeletal muscle in severely obese subjects. Am J Physiol Endocrinol Metab. 2006;290(5):E961-E967.
30. Hayashi N. Guidelines for the Diagnosis and Management of Nonalcoholic Steatohepatitis and Nonalcoholic Fatty Liver Disease. Tokyo, Japan: Bunkodo; 2006. Japanese.

31. Donnelly JE, Blair SN, Jakicic JM, Manore MM, Rankin JW, Smith BK. American College of Sports Medicine Position Stand. Appropriate physical activity intervention strategies for weight loss and prevention of weight regain for adults. Med Sci Sports Exerc. 2009;41(2): 459-471.

\section{Publish your work in this journal}

Therapeutics and Clinical Risk Management is an international, peerreviewed journal of clinical therapeutics and risk management, focusing on concise rapid reporting of clinical studies in all therapeutic areas, outcomes, safety, and programs for the effective, safe, and sustained use of medicines. This journal is indexed on PubMed Central, CAS,
EMBase, Scopus and the Elsevier Bibliographic databases. The manuscript management system is completely online and includes a very quick and fair peer-review system, which is all easy to use. Visit http://www.dovepress.com/testimonials.php to read real quotes from published authors.

Submit your manuscript here: http://www.dovepress.com/therapeutics-and-clinical-risk-management-journal 